\title{
Application of low-dose dual-source computed tomography angiography in children with complex congenital heart disease
}

\author{
XIAN-FENG CHEN, FAN JIANG, LIN LI, YAN CHEN, XIN CHEN, \\ YAN-YAN JIANG, LI XIANG and XIAO-JING MA
}

Department of Radiology, Wuhan Asia Heart Hospital, Wuhan, Hubei 430000, P.R. China

Received October 11, 2015; Accepted July 21, 2016

DOI: $10.3892 /$ etm.2017.4591

\begin{abstract}
The objective of the present study was to evaluate image quality and radiation dosage using a low-dose prospectively electrocardiogram (ECG)-gated computed tomography (CT) protocol for dual-source angiography in children with complex congenital heart disease. A total of 206 patients with complex congenital heart disease were equally assigned into two groups at random. The children in group A underwent low-dose retrospective ECG-gated CT scanning with an ECG-pulsing technique, and group B underwent prospective ECG-gated scanning with an ECG-pulsing technique. Radiation dose volume computed tomography dose index (CTDI vol), dose length product (DLP) and effective dose (ED) were recorded after scanning. Raw data were transferred to workstations for post-processing, diagnosis, grading, comparison with intra-operation findings or cardiac catheterisation, and the coincidence, false negative rate and misdiagnosis rates of groups $\mathrm{A}$ and $\mathrm{B}$, respectively, were subsequently recorded. The results of the present study indicated that the height, age and weight of the children in the two groups exhibited no significant differences. The image quality of group A was graded as 3.94 \pm 0.08 , whereas the grade for the image quality in group B was $4.05 \pm 0.08$; no significant difference was detected. The coincidence rates of groups A and B were 89.37 and $88.48 \%$, respectively; the false negative rates of groups A and B were 9.66 and $10.60 \%$, respectively; the misdiagnosis rates of groups A and B were 0.97 and $0.92 \%$. No significant differences between the two groups were detected. The CTDI value of group A was $3.24 \pm 1.62 \mathrm{mGy}$, the DLP value was $47.53 \pm 33.28 \mathrm{mGy} \cdot \mathrm{cm}^{2}$, the ED value was $0.93 \pm 0.42 \mathrm{mSv}$. By contrast, the CTDI value of group B was $2.27 \pm 0.94 \mathrm{mGy}$, the DLP value was $(27.03 \pm 17.64) \mathrm{mGy} \cdot \mathrm{cm}^{2}$, and the ED value was $0.53 \pm 0.23 \mathrm{mSv}$. Significant differences were detected between the two groups (CTDI $t=5.287$, $\mathrm{P}<0.05$; DLP
\end{abstract}

Correspondence to: Dr Xiao-Jing Ma, Department of Radiology, Wuhan Asia Heart Hospital, 753 Jinghan Road, Jianghan, Wuhan, Hubei 430000, P.R. China

E-mail: 1256760455@qq.com

Key words: radiation, electrocardiographic-pulsing technique, children with congenital heart disease $\mathrm{t}=5.523, \mathrm{P}<0.05 ; \mathrm{ED} \mathrm{t}=8.497, \mathrm{P}<0.05)$, and the radiation dose of group B was markedly decreased, compared with group A. In conclusion, the present study demonstrated that prospectively ECG-gated scanning of dual-source CT is an effective method of examination for dose reduction in children with congenital heart disease without impairment of image quality, which suggests that this protocol may be suitable for future application and dissemination.

\section{Introduction}

Complex congenital heart disease (CHD), which is related to cardiovascular malformations due to abnormal embryonic development, is one of the most common cardiovascular diseases. The incidence of CHD is $0.4-0.8 \%$ in newborns, of which $60 \%$ succumb to CHD during their first year (1). Therefore, CHD is a fatal defect that lowers the life quality of young children (2). As China has the highest birth rate in the world (3), early diagnosis and timely surgical treatment is extremely important for reducing the mortality rate of $\mathrm{CHD}$. Preoperative examination of CHD is conducted by echocardiography, cardiac catheterisation angiography, magnetic resonance imaging (MRI) and computed tomography. Ultrasound cardiograms are convenient and non-invasive, but the vessels outside the heart cannot be displayed clearly $(4,5)$. Cardiac catheterisation angiography is invasive for patients. MRI provides a non-invasive alternative and avoids ionizing radiation exposure, although the examination time of MRI is long, and it is not suitable for children (6). Dual-source computed tomography (DSCT) is a non-invasive technique with high temporal resolution and spatial resolution that is helpful for diagnosis, postoperative effect prediction and follow-up studies $(7,8)$.

The present study aimed to evaluate image quality and radiation dosage using a low-dose prospectively electrocardiogram (ECG)-gated CT protocol for DSCT in children with complex congenital heart disease. From January 2013 to July 2013, a specific scanning schedule was introduced to reduce the radiation dosage in order to limit unnecessary exposure to radiation in children with CHD.

\section{Materials and methods}

Patients. Low-dose protocols were approved by the Ethics Committee of Wuhan Asia Heart Hospital (Wuhan, China). 
During the period from January 2013 to July 2013, 206 children were initially diagnosed with complex congenital heart diseases, which were confirmed by ultrasound cardiogram. To clarify the intracardiac and extracardiac abnormities, patients underwent cardiac CT scanning prior to surgery. These 206 cases, including 105 males and 101 females with an age range of 1-142 months, a mean age of $22.21 \pm 2.89$ months, a mean height of $76.54 \pm 1.88 \mathrm{~cm}$ and a mean weight of $9.76 \pm 0.49 \mathrm{~kg}$, were equally and randomly classified into two groups according to the registered number. Children in group A underwent low-dose prospective ECG-gated scanning, whereas those in group B received retrospective ECG-gated scanning on a DSCT scanner. Informed consent was obtained from the parents of all children enrolled in the present study.

Scanning protocol. Patients were scanned with a DSCT scanner (Siemens Somatom Definition Flash CT; Siemens AG, Munich, Germany). All patients remained in sinus rhythm before examination without receiving a $\beta$-blocker. An intravenous catheter was placed into an antecubital fossa vein or femoral vein in each patient. Short-acting anesthesia (propofol, 1-2 mg/kg; Frsenius Kabi Deutschland GmbH, Germany) was administered to uncooperative patients according to their respective weights. Patients were provided with a lead apron to cover body parts that were not to be scanned to ensure radiation protection. All patients received a nonionic low-osmolality contrast agent Visipaque 320 (iodixanol; GE Healthcare Life Sciences, Chalfont, UK) injection according to their weight $(1.5-2.5 \mathrm{ml} / \mathrm{kg})$ at an injection velocity of $0.7-2.7 \mathrm{ml} / \mathrm{sec}$. A contrast agent dose of $2.0 \mathrm{mg} / \mathrm{kg}$ is typically used. A double tube high pressure syringe (Medrad, Inc., Warrendale, PA, USA) was applied for biphasic injection: $75 \%$ contrast and $25 \%$ saline were injected simultaneously in the first phase, and 6-10 $\mathrm{ml}$ saline was injected at the same velocity in the second phase to eliminate artifacts in the superior vena cava and right atrium (9).

The scanning range of the heart included the area from the thoracic inlet to $1 \mathrm{~cm}$ below the diaphragm, and the scanning direction was set from superior to inferior. The following imaging parameters were used: $80 \mathrm{kV}$ automatic tube current modulation technique (caredose); rotation time, $0.28 \mathrm{sec}$; detector array, $128 \times 0.6 \mathrm{~mm}$; and slice thickness, $0.75 \mathrm{~mm}$. Epigastric CT scanning was carried out following heart scanning to rule out situs inversus viscerum and anomalous pulmonary venous connection. Scanning range was set from the diaphragm to the inferior pole of the kidney.

Scanning methods. Different scanning methods were applied to the children in the two groups. Retrospective ECG-gated helical was used in group A (10), with a continuous volume scan adjusted to an exposure window of $35-75 \%$ of the cardiac cycle. Prospective ECG-triggered axial coronary CTA was used in group B $(11,12)$ in a step-and-shoot scan mode, which adjusted the exposure time between $40-70 \%$ of the cardiac cycle. Radiation dose volume CT dose index (CTDI vol), dose length product (DLP) and effective dose (ED), which is calculated as $\mathrm{ED}=\mathrm{K} x \mathrm{DLP}$, were recorded after scanning. As previously demonstrated, the values of the coefficient $\mathrm{K}$ are variable at different ages (13).
Following post-processing of the raw data, the optimal images were captured at both systole and diastole, with a thickness of $0.75 \mathrm{~mm}$ and a convolution kernel of B26. The imaging mode included maximum intensity projection, multi-plane reconstruction and volume representation amongst others. Diagnosis was compared with intraoperative findings or cardiac catheterisation, and the coincidence, false negative rate and misdiagnosis rates of groups A and B were subsequently recorded.

\section{Assessments of image quality}

Objective evaluation. Regions of interest (ROIs) with a length $>1 \mathrm{~cm}$ were drawn on the right atrium, right ventricle, left atrium, left ventricle, ascending aorta $(1 \mathrm{~cm}$ below the tracheal carina), descending aorta and main pulmonary artery during diastole. ROIs were placed at the center of each region to confirm the uniformity of density and improve measurement performances (14).

Subjective evaluation. Raw data were transferred to workstations for post-processing. Two radiologists subsequently assessed the image quality at post-processing workstations using a 5-point scale (15): (i) 5-point, excellent; (ii) 4-point, good; (iii) 3-point, moderate; (iv) 2-point, suboptimal; and (v) 1-point, unacceptable.

Diagnostic results. A total of 82 children in group A underwent surgery, of which two underwent transcatheter occlusion and one underwent right catheterisation angiography. In group B, 86 children underwent surgery, of which two underwent transcatheter occlusion and two underwent right catheterisation angiography. Intraoperative or cardiac catheterisation findings were subsequently compared with the CT results.

Statistical analysis. Statistical analysis was performed using SPSS 19.0 statistical software (IBM SPSS, Armonk, NY, USA). One-sample Kolmogorov-Smirnov test was used to present statistical data in a normal distribution. Student's $t$-test analysis was performed to determine difference among age, height, weight tube current, image quality, mean value of ROIs, CTDI, DLP and ED factors between two groups. $\mathrm{P}<0.05$ was considered to indicate a statistically significant difference.

\section{Results}

All 206 children in the two groups suffered from malformation of cardiac structure, anomalous with great vascular and malformation of trachea and visceral organs (Table I). In total, 476 different malformations were diagnosed by DSCT, including: Tetralogy of Fallot (Fig. 1), double-outlet right ventricle (DORV), pulmonary atresia, anomalous pulmonary venous connection, transposition of the great arteries, and coarctation of the descending aorta (Table II). There were no significant differences identified in the image quality scores between the two groups (Table III).

In group A, 237 types of malformation were detected, including: Atrial septum defects $(n=25)$; ventricular septum defects $(n=26)$; patent ductus arteriosus $(n=19)$; anomalies of the morphological structure of the pulmonary valve $(n=17)$; 
Table I. Characteristics of the children in the two groups.

\begin{tabular}{lccc}
\hline Group & Age (months) & Height $(\mathrm{cm})$ & Weight $(\mathrm{kg})$ \\
\hline Group A & $22.2 \pm 2.9$ & $76.6 \pm 1.9$ & $9.76 \pm 0.49$ \\
Group B & $22.9 \pm 2.7$ & $77.9 \pm 1.8$ & $10.16 \pm 0.56$ \\
P-value & 0.851 & 0.617 & 0.459 \\
\hline
\end{tabular}

The patients' ages, heights, weights and number of deformations exhibited no statistically significant differences $(\mathrm{P}>0.05)$. Data are presented as the mean \pm standard deviation.

anomalous vena caval connection $(\mathrm{n}=13)$; pulmonary stenosis or anomalous origin of the arterial branch $(\mathrm{n}=10)$; anomalous origin of the coronary artery $(n=8)$; heteroplasia of the aortic arch $(n=7)$; patent oval foramen $(n=5)$; bronchial stenosis or anomalies of the morphological structure $(n=4)$; aberrant subclavian artery $(\mathrm{n}=5)$; situs viscera inversus, asplenia or polysplenia $(\mathrm{n}=3)$; disorders of the mitral valve $(\mathrm{n}=2)$; cor triatriatum $(\mathrm{n}=1)$; unroofed coronary sinus syndrome $(\mathrm{n}=1)$; atrioventricular discordance $(\mathrm{n}=1)$; and mirror image dextrocardia $(\mathrm{n}=1)$.

In group B there were 239 types of malformation detected, including: Atrial septum defects $(n=29)$; ventricular septum defects ( $\mathrm{n}=32)$; patent ductus arteriosus ( $\mathrm{n}=14)$ (Fig. 2); anomalies of the morphological structure of the pulmonary valve $(\mathrm{n}=17)$; anomalous vena caval connection $(\mathrm{n}=8)$; pulmonary stenosis $(n=7)$; anomalous origin of the coronary artery $(n=7)$; heteroplasia of the aortic arch $(n=9)$; patent oval foramen $(\mathrm{n}=8)$; bronchial stenosis or anomalies of the morphological structure $(n=5)$; aberrant subclavian artery $(n=9)$; asplenia with situs viscera inversus $(\mathrm{n}=1)$; unroofed coronary sinus syndrome $(n=1)$; and hepatic carcinoma $(n=1)$.

Among all the cases in groups A and B, there were 175 children (424 types of malformation) who underwent therapeutic surgery or cardiac catheterisation to confirm the diagnosis of CT. In group A, foramen ovale patent $(n=16)$, atrial septum defects $(n=3)$ and muscular part of ventricular septum defect $(n=1)$ were not detected, and two normal ventricular septums were misdiagnosed as having ventricular septal defects. In group B, the following were not detected: Foramen ovale patent $(n=17)$, atrial septum defect $(n=4)$, patent ductus arteriosus $(n=2)$ and muscular part of ventricular septum defects $(n=2)$. Two normal ventricular septal were misdiagnosed as being the muscular part of a ventricular septum defect. In group A, the coincidence rate of $\mathrm{CT}$ and clinical diagnoses was $89.37 \%$, the rate of missed diagnosis was $9.66 \%$, and the false positive rate was $0.97 \%$. In group B, the coincidence rate of $\mathrm{CT}$ and clinical diagnoses was $88.48 \%$, the rate of missed diagnosis was $10.60 \%$, and the false positive rate was $0.92 \%$. No significant differences were identified between the two groups. No statistical differences in the mean values of ROI and tube current were identified between the two groups (Table IV).

\section{Discussion}

During the important stage of growth and development, between birth and twelve years of age, children are 10 times more sensitive to radiation than adults (16); therefore, the
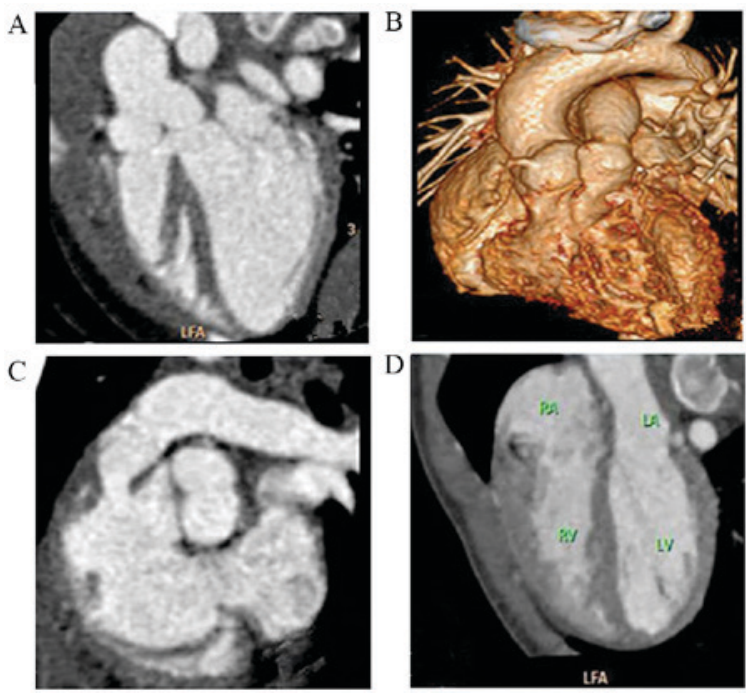

Figure 1. A case in group A (female; age, 13 months; height, $75.5 \mathrm{~cm}$; weight, $9 \mathrm{~kg}$ diagnosed with Tetralogy of Fallot. (A) The degree of aortic override was $50 \%$. (B) Volume rendring demonstrated an aortic root directly above the septal defect; (C) maximum intensity projection showed narrowing of the right ventricular outflow tract, which was indicative of a ventricular septal defect. (D) Atrium and ventricle was normal connection.
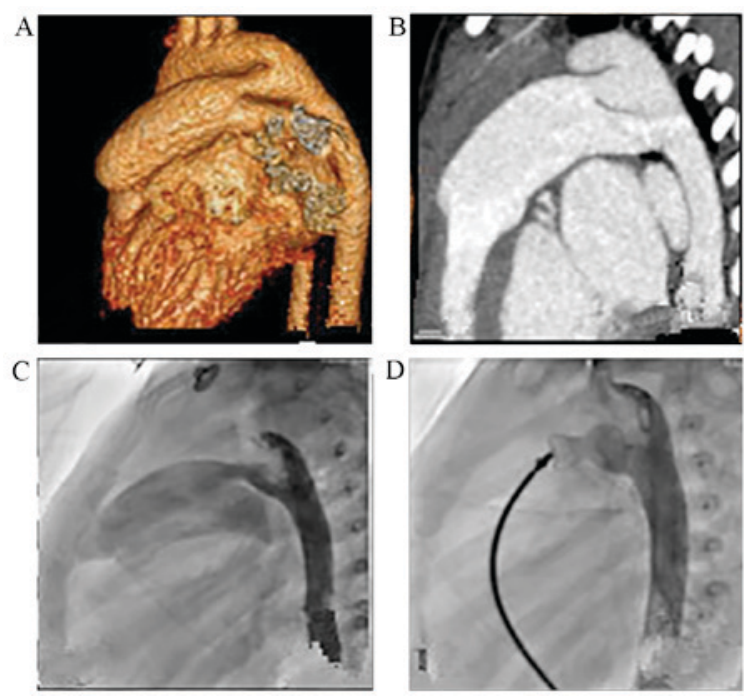

Figure 2. A case in group B (female; age, 15 months; height, $75 \mathrm{~cm}$; weight $9 \mathrm{~kg}$ ) diagnosed with patent ductus arteriosis. (A) Volume rendering, (B) maximum intensity projection and $(\mathrm{C})$ digital subtraction angiography imaging demonstrated an abnormal tubular structure between the descending aorta and main pulmonary artery. (D) The shunt was detected after device closure.

importance of radiation protection cannot be overemphasised. At present, the main method used to reduce radiation dosage is auto tube current modulation and the restriction of tube voltage (17-19). The present study responded to the growing concern over the radiation doses administered to children and applied a novel scanning method. According to the two gating techniques used, children were equally classified into groups A and B (20). In group A, retrospective ECG-gating with an ECG-pulsing technique was applied and the exposure window of this group was adjusted to $35-75 \%$ of the cardiac cycle; whereas the exposure window of group B was adjusted to $40-70 \%$. The mean ED of group A was $0.93 \pm 0.41 \mathrm{mSv}$ and the 
Table II. Main deformities diagnosed by dual-source computed tomography in patients with complex congenital heart disease in the two groups.

\begin{tabular}{lcc}
\hline Disease deformities & Group A (n) & Group B (n) \\
\hline Tetralogy of Fallot & 36 & 18 \\
Double outlet right ventricle & 7 & 13 \\
Coarctation of the descending aorta & 6 & 8 \\
Pulmonary atresia & 5 & 3 \\
Patent ductus arteriosus & 5 & 6 \\
Endocardial cushions defect & 3 & 1 \\
Anomalous pulmonary venous connection & 3 & 10 \\
Single atrium & 1 & 2 \\
Transposition of the great arteries & 1 & 5 \\
Single ventricle & 1 & 2 \\
Right coronary artery fistula to left ventricle & - \\
Right coronary artery fistula to right ventricle & 1 \\
Double-chambered right ventricle & 1 \\
Anomalous origin of coronary artery from pulmonary artery & - \\
Anomalous origin of right pulmonary artery from ascending aorta & - & - \\
Aneurysm of the membranous ventricular septum & 1 & - \\
Interruption of aortic arch & - \\
Tricuspid atresia & - & 1 \\
\hline
\end{tabular}

Table III. Image quality scores of the two groups.

\begin{tabular}{lcccccc}
\hline & \multicolumn{5}{c}{ Grade } & Image quality scores \\
\cline { 2 - 6 } & 5 & 4 & 3 & 2 & 1 & 0 \\
\hline Group A & 23 & 56 & 19 & 5 & 0 & $3.94 \pm 0.08$ \\
Group B & 31 & 48 & 22 & 2 & $-05 \pm 0.08$ \\
P-value & - & - & - & - & - & 0.324 \\
\hline
\end{tabular}

There were no significant differences identified in the image quality scores between the two groups. Data are presented as the mean \pm standard deviation.

Table IV. Mean values of ROI, tube current and radiation dose in the two groups.

\begin{tabular}{|c|c|c|c|c|c|}
\hline Group & Mean ROI (Hu) & Tube current (mAs) & CTDI (mGy) & DLP (mGy.cm) & $\mathrm{ED}(\mathrm{mSv})$ \\
\hline Group A & $476.2 \pm 4.96$ & 80.65 & 3.24 & 47.53 & 0.93 \\
\hline Group B & $470.9 \pm 4.32$ & 84.11 & 2.27 & 27.03 & 0.53 \\
\hline P-value & 0.755 & 0.327 & $<0.001$ & $<0.001$ & $<0.001$ \\
\hline
\end{tabular}

No statistical differences in the mean values of ROI and tube current were identified between the two groups. For the two groups, CTDI, $\mathrm{t}=5.287(\mathrm{P}<0.001)$; DLP, $\mathrm{t}=5.523,(\mathrm{P}<0.001)$; and $\mathrm{ED}, \mathrm{t}=8.497(\mathrm{P}<0.001)$. Radiation doses in group B were significantly $(75.03 \%)$ lower than those in group $\mathrm{A}(\mathrm{P}<0.05)$. Data are presented as the mean \pm standard deviation. ROI, region of interest; CTDI, computed tomography dose index; DLP, dose length product; ED, effective dose.

radiation dose was reduced by $75 \%$. Therefore, the radiation dose remained inadequately controlled due to the continuous scanning.

The mean ED of group B was $0.53 \pm 0.23 \mathrm{mSv}$, which is markedly lower than the standard CT scanning dose by virtue of the step-and-shoot mode (21). For patients with situs viscera inversus, the final phase of the scan protocol was set as a low-dose abdominal scan in order to detect any structural abnormalities of the abdominal organs and arteriovenous malformation. Both groups A and B underwent abdominal scans with Siemens Care Dose technology $(70 \mathrm{kV}$; 20-30 mAs), which administers a very low radiological dose 
with a DLP of $4-5 \mathrm{mGy} \cdot \mathrm{cm}$. DLP is the product of the CTDI vol and X-ray scan length of subjects, ED is the X-ray dose that causes biological damage to subjects, the $\mathrm{K}$-value is the conversion coefficient of DLP and ED $[\mathrm{ED}(\mathrm{mSv})=\mathrm{K}$ $(\mathrm{mSv} /[\mathrm{mGy} \cdot \mathrm{cm}]) \times$ DLP $(\mathrm{mGy} \cdot \mathrm{cm})](13)$, and the conversion coefficient varies based on the patient's age and body part scanned. In the present study, according to the AAPM Report 96 (2008) (22), in chest routine scans, the K-values were as follows: Newborn, 0.039; 1-year-old, 0.026; 5-year-old, 0.018; 10-year-old, 0.013; and adult, 0.014 .

The most effective method of reducing the radiation dosage typically comes at the cost of impairing image quality. In the present study, the data showed that there was no significant difference in image quality between the two groups.

By analyzing the surgical findings, one can observe that defects $<0.5 \mathrm{~mm}$ were easily misdiagnosed or undetected since the resolution was $>0.5 \mathrm{~mm}$. In the present study, patent foramen ovale, atrial septum defects and ventricular septum defects $<0.5 \mathrm{~mm}$ were misdiagnosed or undetected. This was predominantly pronounced in cases with patent foramen ovale as this type of defect typically appears to mimic a flap valve, only opening during certain conditions when there is increased pressure inside the chest; therefore, this defect is consistently detected during exploratory surgery but remains difficult to diagnose via CT scanning (23). In addition, two patent ductus arteriosi were left undetected in group B, whose sizes were $<1 \mathrm{~mm}$.

Typically, the three main factors influencing the radiation dose in patients are CTDI, DLP and ED $(24,25)$. By comparing these factors between the two groups, significant differences were detected. Furthermore the image quality of group B was not significantly different to that of group A, demonstrating that prospective ECG-gated CT with ECG-pulsing is effective at reducing the radiation dosage and avoids the impairment of image quality.

To optimise the scanning phase, previous studies $(20,26)$ have suggested that the exposure window should be adjusted to $40 \%$ of systole as the majority of children with CHD exhibit rapid heart rates. As such, cardiac function cannot be evaluated without a cardiac systole during scanning (27). In the present study, scanning was performed during the double phase of diastole and systole; therefore, the diameter of the defect or stricture could be measured accurately and cardiac function was quantitatively analysed (28-31).

Proper usage of contrast medium is a vital procedure in cardiac angiography and bolus tracking is one of the most common methods used to visualise vessels clearly. The volume of contrast is tracked using an ROI at a certain level and when the CT reaches this level the images are acquired at a rate as fast as the contrast moves through the vessels (32). Complex $\mathrm{CHD}$, however, altered the haemodynamics to varying degrees due to the presence of extracardiac deformities, ventriculoarterial connections, alterations in ventricular volume and rapid heart rates. In particular, the DORV, transposition of the great arteries, pulmonary atresia, tricuspid atresia and other complex deformations cause large shunting, which seriously affected the image quality (33).

The bolus injection used in the present study has limitations. Firstly, it may cause a banding artifact in the superior vena cava and right atrium when normal saline is not filling into the right atrium as soon as possible. Secondly, there is not enough contrast medium in the left heart system, which may result in faint development, and the poor development of the descending aorta is one of the factors that may result in small diameter patent ductus arteriosus or major aorta pulmonary collateral arteries being left undetected. Thirdly, the malformation of the atrium and the structure of the tricuspid valve cannot be accurately identified.

Directly delayed contrast-enhanced scanning was applied in the present study. Prior to scanning, medical history was collected and ECG was performed, and a double tube high-pressure syringe was subsequently applied for biphasic injection. According to the heart rate of the patients and the type of malformation, different integral doses, velocities, and scanning ranges were selected. Following a delay of 20-25 sec, the scan was processed, which lasted 2-6 sec.

The scanning method used in the present study guaranteed the excellent demonstration of the cardiovascular structure without artifacts and with proper usage of the contrast medium (34), leading to the reduced risk of renal injury. Therefore, the present study showed that proper contrast medium combined with low $\mathrm{kV}$ scanning enables the accurate diagnosis of complex CHD. Furthermore, all the patients successfully completed the examination without any adverse reactions.

Subsecond scanning and isotropic imaging of 64-multi-slice CT (MSCT) facilitates the clinical application of coronary angiography. In combination with the high accuracy of echocardiogram for the assessment of cardiac structure, 64-MSCT was preliminarily applied in the diagnosis of complex CHD. However, due to the restriction of time resolution, CHD patients whose heart rates were $>100 \mathrm{bpm}$ required retrospective $\mathrm{ECG}$-gated $\mathrm{CT}$ for scanning so that the scan time was prolonged and the scan range was limited. As a result, the radiation dosage increases, which is harmful to children's growth and development (35).

On account of the detector array, 320-MSCT can be used to cover the heart with a routine scan (36). Patients with slow heart rates are able to complete the heart examination during one cardiac period, whereas patients with faster heart rates require multiple cardiac periods to complete the scan, and the risk of multiple exposures is increased. For the images of patients whose heart rates exceed $100 \mathrm{bpm}$, extensive post-processing is required. As previously described, the mean effective radiation dose of $\sim 1.17 \mathrm{mSv}$ is higher than the mean radiation dose applied in this study $(25,37)$. Notably, 320-MSCT allows CT angiography examination to be performed at high-pitch values of 3.4, and at a scanning rate of up to $43 \mathrm{~cm} / \mathrm{sec}$ (38). Additionally, the advantages include an extremely short exposure time, a low radiation dose, and scanning is unaffected by respiratory rate. Therefore, fast heart rates and arrhythmia remain challenging during 320-MSCT (39). Prospective ECG-gating is an ideal method that is applicable for patients with rapid heart rates or arrhythmia. Advances in scientific research concerning radiation doses have shown that, in a chest scan and angiocardiography, the radiation dose of DSCT was only $24 \%$ of the traditional $\mathrm{CT}$, which is a notable development in overcoming the restriction of radiation dosage (40).

High time resolution $(75 \mathrm{msec}$ ) of DSCT overcomes the limitations of heart rate during CT scanning, and prospective 
ECG-gated CT scanning is a more suitable method for complex CHD patients with tachycardia or tachypnoea. Moreover, the ECG-pulsing technique is able to greatly reduce the radiation dose without impairing image quality. Therefore, prospective ECG-gated CT scanning with an ECG-pulsing technique, low tube voltage and automatic tube current modulation technique may be a feasible protocol for reducing radiation dosages.

In conclusion, ECG is the preferred method for the diagnosis of complex CHD; however, CT scanning can provide additional useful information that may increase the diagnosis rate prior to surgery. DSCT has an unbeatable advantage in its scanning pattern, which optimises the protocol according to the clinical features of the patient and disease to effectively reduce the radiation dosage to a level that is reasonable and achievable.

\section{Acknowledgements}

This study was supported by Wuhan Research Funded Projects (grant no. WX13Z04).

\section{References}

1. Sayin Kocakap BD, Sanli C, Cabuk F, Koc M and Kutsal A: Association of MTHFR A1298C polymorphism with conotruncal heart disease. Cardiol Young 25: 1326-1331, 2015.

2. Holst KA, Dearani JA, Burkhart HM, Connolly HM, Warnes CA, $\mathrm{Li} \mathrm{Z}$ and Schaff HV: Risk factors and early outcomes of multiple reoperations in adults with congenital heart disease. Ann Thorac Surg 92: 122-130, 2011.

3. Flohr TG, Leng S, Yu L, Aiimendinger T, Bruder H, Petersilka M, Eusemann CD, Stierstorfer K, Schmidt B and McCollough CH: Dual-source spiral CT with pitch up to 3.2 and $75 \mathrm{~ms}$ temporal resolution: Image reconstruction and assessment of image quality. Med Phys 36: 5641-5653, 2009.

4. Pushparajah K, Miller OI and Simpson JM: 3D echocardiography of the atrial septum: Anatomical features and landmarks for the echocardiographer. JACC Cardiovasc Imaging 3: 981-984, 2010.

5. Song BG, Park SW, Lee SC, Choi JO, Park SJ, Chang SA, Oh JK and Yang JH: Real-time 3D TEE for multiperforated interatrial septum. JACC Cardiovasc Imaging 3: 1199, 2010.

6. Fratz S, Chung T and Gerald F Greil GF, Samyn MM, Taylor AM, Buechel ER, Yoo SJ and Powell AJ: Guidelines and protocols for cardiovascularmagnetic resonance in children and adults withcongenital heart disease: SCMR expert consensus group on congenital heart disease. J Cardiovasc Magn Reson 15: 1, 2013.

7. Al-Mousily F, Shifrin RY, Fricker FJ, Feranec N, Quinn NS and Chandran A: Use of 320-detector computed tomographic angiography for infants and young children with congenital heart disease. Pediatr Cardiol 32: 426-432, 2011.

8. Sun XH, Sun ZG, Li Z, Shi Z, Wang L, Sheng H, Wu T and Li C: The study of dual source computed tomography angiography in the evaluation of extracardial anomalies in patients with congenital heart disease. J Med Imaging 20: 667-671, 2010.

9. Khatri S, Varma SK, Khatri P and Kumar RS: 64-slice multidetector-row computed tomographic angiocardiography for evaluating congenital heart disease. Pediatr Cardiol 29: 755-762, 2008 .

10. Hollingsworth CL, Yoshizumi TT, Frush DP, Chan FP, Toncheva G, Nguyen G, Lowry CR and Hurwitz LM: Pediatric cardiac-gated CT angiography: Assessment of radiation dose. AJR Am J Roentgenol 189: 12-18, 2007.

11. Liu GR and Li YC (eds): Dual-source definition flash CT in diagnosis of cardiovascular and cerebrovascular disease. People's Medical Publishing House, Beijing, 2013.

12. Sabarudin A, Sun Z and Yusof AK: Coronary CT angiography with single-source and dual-source CT: Comparison of image quality and radiation dose between prospective ECG-triggered and retrospective ECG-gated protocols. Int J Cardiol 168: 746-753, 2013
13. Thomas KE and Wang B: Age-specific effective doses for pediatric MSCT examinations at a large children's hospital using DLP conversion coefficients: A simple estimation method. Pediatr Radiol 38: 645-656, 2008.

14. Heyer CM, Mohr PS, Lemburg SP, Peters SA and Nicolas V: Image quality and radiation exposure at pulmonary CT angiography with 100 -or $120-\mathrm{kVp}$ protocol: Prospective randomized study. Radiology 245: 577-583, 2007.

15. Pache G, Grohmann J, Bulla S, Arnold R, Stiller B Schlensak C, Langer M and Blanke P: Prospective electrocardiography-triggered CT angiography of the great thoracic vessels in infants and toddlers with congenital heart disease: Feasibility and image quality. Eur J Radiol 80: e440-e445, 2011.

16. Donnelly LF: Reducing radiation dose associated with pediatric CT by decreasing unnecessary examination. AJR Am J Roentgenol 184: 655-657, 2005.

17. Zhang J, Yang M, Mo XM, Jin JY, Liu B, Li LL and Teng GJ: Impact of different tube voltage protocols on image quality and radiation dosage for pediatric 64-slice cardiovascular CT angiography. Chin J Med Imaging Technol 28: 1213-1217, 2012.

18. Kalra MK, Maher MM, Toth TL, Schmidt B, Westerman BL, Morgan HT and Saini S: Techniques and applications of automatic tube current modulation for CT. Radiology 233:649-657, 2008.

19. Park CK, Choo KS, Jeon UB, Baik SK, Kim YW, Kim TU, Kim CW, Jeong YJ, Jeong DW and Lim SJ: Image quality and radiation dose of 128-slice dual-source CT venography using low kilovoltage combined with high-pitch scanning and automatic tube current modulation. Int J Cardiovasc Imaging 29: (Suppl 1) 47-51, 2013

20. Nie P, Wang X, Cheng Z, Ji X, Duan Y and Chen J: Accuracy, image quality and radiation dose comparison of high-pitch spiral and sequential acquisition on 128-slice dual-source CT angiography in children with congenital heart disease. Eur Radiol 22: 2057-2066, 2012.

21. Klass O, Walker M, Siebach A, Stuber T, Feuerlein S, Juchems M and Hoffmann MH: Prospectively gated axial CT coronary angiography: Comparison of image quality and effective radiation dose between 64- and 256-slice CT. Eur Radiol 20: 1124-1131, 2010.

22. Shrimpton PC, Hillier MC, Lewis MA and Dunn M: National survey of doses from CT in the UK: 2003. Br J Radiol 79: 968-980, 2014.

23. Yang SY (ed): Pediatric cardiology. People's Medical Publishing House, Beijing, 2009.

24. Xu J, Zhao H, Wang X, Bai Y, Liu L, Liu Y, Wei M, Li J and Zheng M: Accuracy, image quality, and radiation dose of prospectively ECG-triggered high-pitch dual-source CT angiography in infants and children with complex coarctation of the aorta. Acad Radiol 21: 1248-1254, 2014.

25. Paul JF, Rohnean A, Elfassy E and Sigal-Cinqualbre A: Radiation dose for thoracic and coronary step-and-shoot CT using a 128-slice dual-source machine ininfants and small children with congenital heart disease. Pediatr Radiol 41: 244-249, 2011.

26. Ren H, Rong Y, Zhang YQ and Hu X: Investigation of coronary angiography by dual-source definition flash CT with multiple scan modes and relative radiation dose. Chin J Radiol Med Prot 32: 329-330, 2012.

27. Cheng Z, Wang X, Duan Y, Wu L, Wu D, Chao B, Liu C, Xu Z, Li $\mathrm{H}$ and Liang F: Low-dose prospective ECG-triggering dual-source CT angiography in infants and children with complex congenital heart disease: First experience. Eur Radiol 20: 2503-2511, 2010.

28. Goo HW and Yang DH. Coronary artery visibility in free-breathing young children with congenital heart disease on cardiac 64-slice CT: Dual-source ECG-triggered sequential scan vs. single-source non-ECG-synchronized spiral scan. Pediatr Radiol 40: 1670-1680, 2010.

29. Ben Saad M, Rohnean A, Sigal-Cinqualbre A, Adler G and Paul JF: Evaluation of image quality and radiation dose of thoracic and coronary dual-source CT in 110 infants with congenital heart disease. Pediatr Radiol 39: 668-676, 2009.

30. Oda S, Utsunomiya D, Funama Y, Awai K, Katahira K, Nakaura T, Yanaga Y, Namimoto T and Yamashita Y: A low tube voltage technique reduces the radiatiun dose at retrospective ECG-gated cardiac computed tomography for anatomical and functional analyses. Acad Radio 18: 991-999, 2011.

31. Hoe J and Toh KH: First experience with 320-row multidetector CT coronary angiography scanning with prospective electrocardiogram gating to reduce radiation dose. J Cardiovasc Comput Tomogr 3: 257-611, 2009. 
32. Szucs-Farkas Z, Schibler F, Cullmann J, Torrente JC, Patak MA, Raible S, Hoppe H, Wyttenbach R, Vock P and Schindera ST: Diagnostic accuracy of pulmonau CT angiograph at low tube voltage: Intraindividual comparison of a normal-dose protocol at $120 \mathrm{kVp}$ and a low-dose protocol at $80 \mathrm{kVp}$ using reduced amount of contrast medium in a simulation study. AJR Am J Roentgenol 197: W852-W859, 2011.

33. Sable C, Foster E, Uzark K, Bjornsen K, Canobbio MM, Connolly HM, Graham TP, Gurvitz MZ, Kovacs A, Meadows AK, et al: Best practices in managing transition to adulthood foradolescents with congenital heart disease: The transition process and medical and psychosocial issues: A scientific statement from the American Heart Association. Circulation 123:1454-1485, 2011.

34. Rybicki FJ, Otero HJ, Steigner ML, Vorobiof G, Nallamshetty L, Mitsouras D, Ersoy H, Mather RT, Judy PF, Cai T, et al: Initial evaluation of coronary images from 320 -detector row computed tomography. Int J Cardiovase Imaging 24: 535-546, 2008.

35. Han BK, Rigsby CK, Hlavacek A, Leipsic J, Nicol ED, Siegel MJ Bardo D, Abbara S, Ghoshhajra B, Lesser JR, et al: Computed tomography imaging in patients with congenital heart disease Part I: Rationale and utility. An Expert Consensus Document of the Society of Cardiovascular Computed Tomography (SCCT) Endorsed by the Society of Pediatric Radiology (SPR) and the. and the North American Society of Cardiac Imaging (NASCI) J Cardiovasc Comput Tomogr 9: 475-492, 2015.
36. Du J, Jiang T, Zhou J, Lv B, Zhang ZQ and Chen W: Application of 320-row detector dynamic volume CT in infants with complicated congenital heart diseases. Chin J Med Imaging Technol 27: 1174-1177, 2011.

37. Tognolini A, Arellano CS, Marfori W, Heidari G, Sayre JW, Krishnam MS and Ruehm SG: Comprehensive low-dose imaging of carotid and coronary arteries with a single-injection dualsource CT angiography protocol. Clinical Radiol 69: 246-253, 2014

38. Li J, Huan Y and Zhao HL, Wang Y, Liu Y, Wei M, Shi $M$ and Zheng $M$ : Comparison of prospective electrocardiography-gating high-pitch mode and without electrocardiography-synchronization high-pitch mode acquisition for the image quality and radiation doses of the aortic using dual-source CT. Chin J Radiol 47: 301-304, 2013.

39. de Broucker T, Pontana F, Santangelo T, Faivre JB, Tacelli N, Delannoy-Deken V, Duhamel A, Remy J and Rémy-Jardin M: Single- and dual-source chest CT protocols: Levels of radiation dose in routine clinical practice. Diagn Interv Imaging 93, $852-858,2012$.

40. Weustink AC, Neefjes LA, Kyrzopoulos S, van Straten M, Neoh Eu R, Meijboom WB, van Mieghem CA, Capuano E, Dijkshoorn ML, Cademartiri F, et al: Impact of heart rate frequency and variabilitv on radiation exposure, image quality, and diagnostic performance in dual-source spiral CT coronary angiography. Radiology 253: 672-680, 2009. 